\title{
Supporting Information \\ Construction of Position-Controllable Graphene Bubbles in Liquid Nitrogen with Assistance of Low-power Laser
}

Xin Zhang, ${ }^{a}$ Haojie Zhang, ${ }^{b}$ Shiwei Cao ${ }^{c}$ Ning Zhang, ${ }^{d}$ Bo Jin, ${ }^{a}$ Zewen Zong, ${ }^{a}$ Zhan Li $^{* a}$ and Ximeng Chen ${ }^{* a}$

\section{CONTENTS}

1 Source of graphene

2 Effect of heat treatment time on graphene film

3 Supplementary AFM images of graphene bubbles

4 Raman spectrum

5 Formation mechanism of the holes

6 Control experiments

7 Co-relation between wrinkles and bubbles

8 Peaks shift

9 Phase transition of nitrogen molecules

10 Calculational details

II Diffusion coefficient

12 Phase transition barrier

13 Descriptions of the supplementary movies

a The School of Nuclear Science and Technology, Lanzhou University, Lanzhou, 730ooo, P.R. China. E-mail: lizhancg@licp.cas.cn; chenxm@lzu.edu.cn.

$b$ School of Physics, Nankai University, Tianjin, 300071, P. R. China.

c Institute of Modern Physics, Chinese Academy of Sciences, Lanzhou, 7300oo, P.R. China.

$d$ School of Physics, Peking University, Beijing, 100871, P.R.China. 
The monolayer graphene on $\mathrm{SiO}_{2}$ used in this work is a commercial product made by chemical vapor phase deposition (CVD) (Nanjing Jicang nanotechnology Co. Ltd, China). Specifically, the monolayer graphene membrane is originally grown on a copper foil. Fast Transfer Graphene technique has been employed by the skilled technicians of Nanjing Jicang nanotechnology Co. Ltd to transfer the monolayer graphene onto $\mathrm{SiO}_{2}$ substrate. In addition, The monolayer graphene on $\mathrm{SiO}_{2}$ has not undergone any heat treatment by Nanjing Jicang nanotechnology Co. Ltd. The size of the graphene purchased is $1 \mathrm{~cm} \times 1 \mathrm{~cm}$.

\section{EFFECT OF HEAT TREATMENT TIME ON GRAPHENE FILM}

In our work, the effect of different heat treatment time on graphene film was studied, and the results are displayed in Fig. Si. As the heat treatment time increases from oh to $12 \mathrm{~h}$, it is found that the graphene film does become thinner. However, the thickness of the graphene film after 3 and 12 heat treatment has no obvious difference (Fig. $\mathrm{S}_{1 \mathrm{~b}}$ and $\mathrm{S}_{\mathrm{I}}$ ). Based on the results of these experiments, $3 \mathrm{~h}$ of heat treatment time is enough in our experiment.
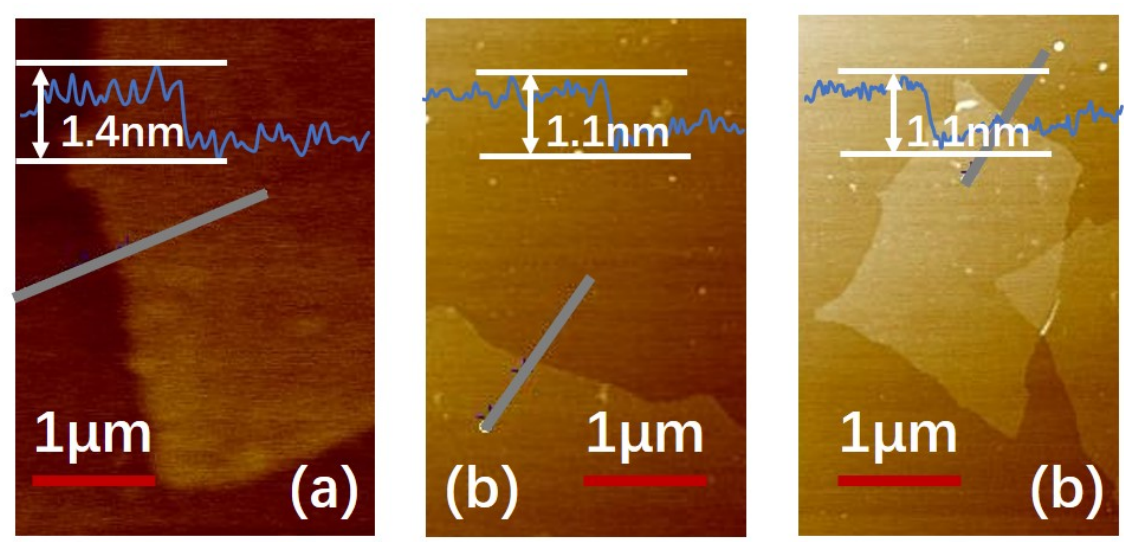

Fig. S 1: AFM images of the graphene films with different heat-treated time. (a) Without heat-treatment; (b) with heat-treatment at $200{ }^{\circ} \mathrm{C}$ for $3 \mathrm{~h}$ under UHV; (c) with heat-treatment at $200{ }^{\circ} \mathrm{C}$ for $12 \mathrm{~h}$ under UHV. 


\section{SUPPLEMENTARY AFM IMAGES OF GRAPHENE BUBBLES}

(a)
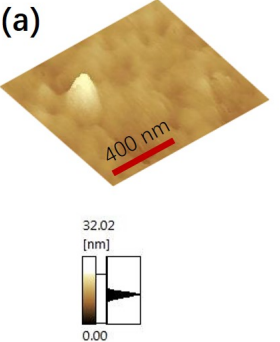

(b)
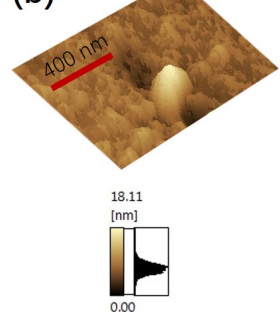

(c)
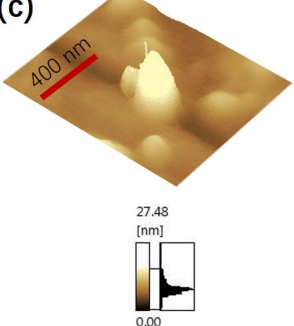

(d)
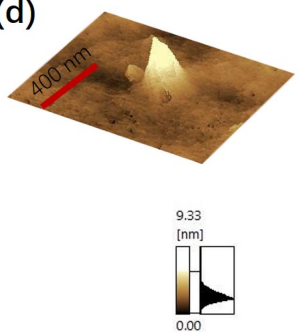

Fig. S 2: AFM images of graphene bubbles. The aspect ratio of (a) 0.12, (b) 0.08 , (c) 0.10 and (d) 0.07.
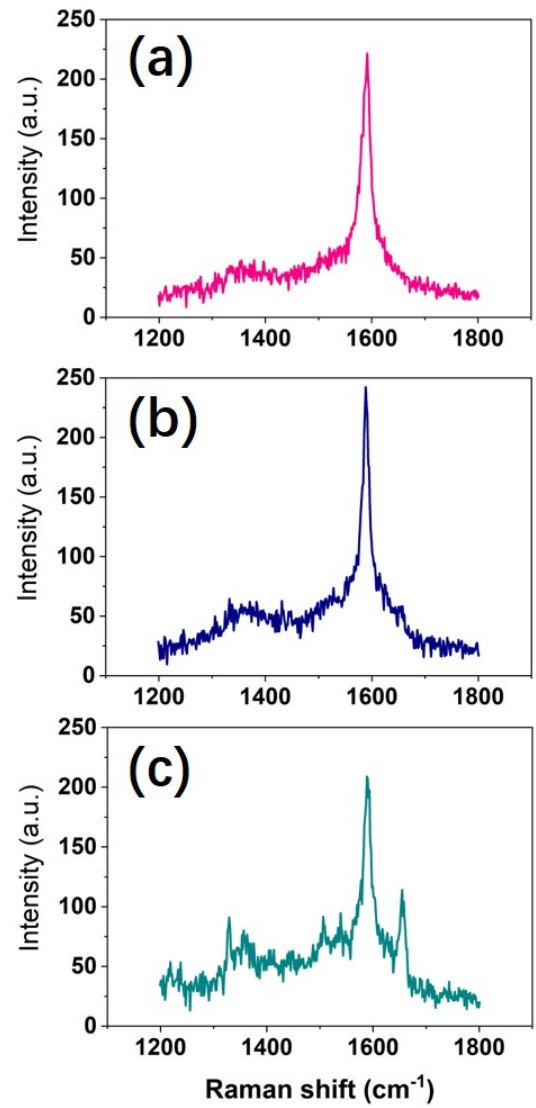

Fig. S 3: Raman spectrum of the three different samples. (a) Pristine Single Layer Graphene on the surface of $\mathrm{SiO}_{2}$ (PSLG@SiO ${ }_{2}$ ); (b) PSLG@SiO ${ }_{2}$ soaked by liquid nitrogen; (c) PSLG@SiO ${ }_{2}$ irradiated by laser in liquid nitrogen. 


\section{FORMATION MECHANISM OF THE HOLES}

First of all, as shown in Fig. S3c, the graphene film irradiated by the laser in liquid nitrogen show us a $\mathrm{D}^{\prime}$ band at $1660 \mathrm{~cm}^{-1}$. The $\mathrm{D}^{\prime}$ band is related to the defects of graphene film. This probably means that after laser irradiation, many $s p^{3}$ hybrid carbon atoms appeared on the graphene film. Secondly, when the graphene film (irradiated by the laser) is exposed to the electron beam of the SEM, it is found that the $s p^{3}$ hybrid carbon atoms of graphene bubbles can be etched by the electron beam. Moreover, the graphene bubbles which were etched by the electron beam would be obviously damaged in an inert environment after 12 days (Fig. $\mathrm{S}_{4} \mathrm{a}$ ). In this case, the larger holes (diameter $\sim 500 \mathrm{~nm}$ ) were not observed. Conversely, the bubbles which were not etched by the electron beam would still maintain its original appearance in an inert atmosphere after 12 days (Fig. $\mathrm{S}_{4} \mathrm{~b}$ ). Thirdly, when the graphene bubbles which etched by electron beam were stored in an atmospheric environment for 12 days, obvious larger holes (diameter $\sim 500 \mathrm{~nm}$ ) will be observed. In summary, the formation mechanism of the holes should be like this: (1) the graphene bubbles were initially modified by the laser power; (2) the lasermodified bubbles were etched by the electron beam, forming small nanopores; (2) small nanopores of the graphene bubbles were etched and oxidized by water molecules and oxygen in the atmosphere, and eventually the larger holes were formed.
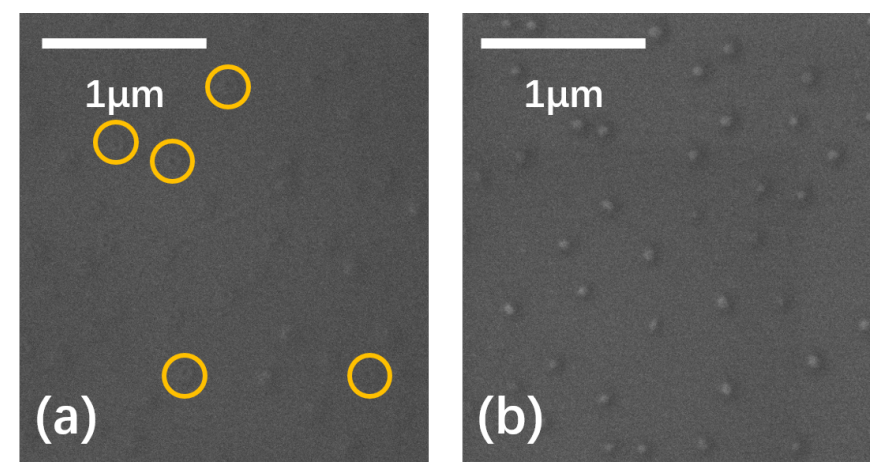

Fig. S 4: SEM images of the graphene bubbles with different treatments. (a) Electron beam etched graphene bubbles stored in an inert environment for 12 days; (b) pristine graphene bubbles stored in an inert environment for 12 days. In order to show the destroyed bubbles more clearly, they are marked with yellow circles in (a).

\section{CONTROL EXPERIMENTS}
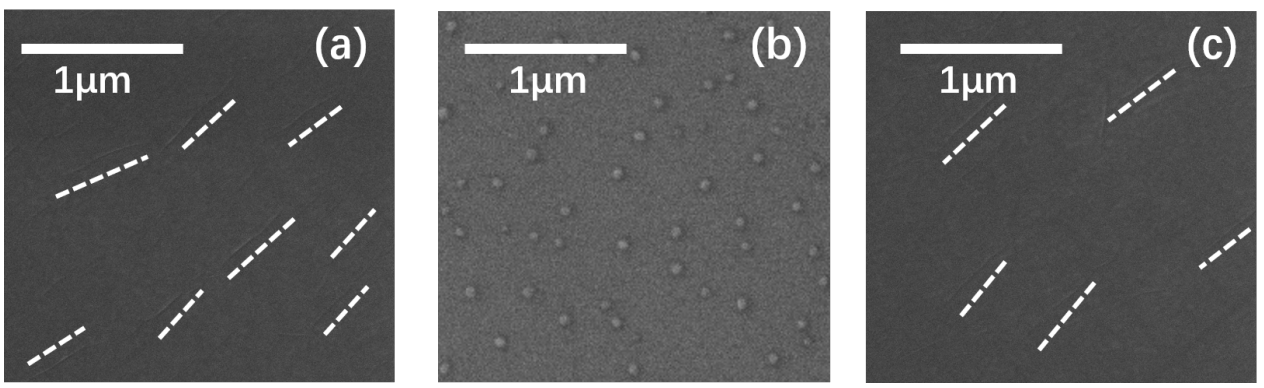

Fig. S 5: SEM images of the three samples under different experimental conditions. (a) Graphene film without laser illumination; (b) graphene film without heat-treatment prior to main experiment; (c) graphene film without heat-treatment and no liquid $\mathrm{N}_{2}$ cooling. In order to show the wrinkles more clearly, a white dotted line is placed beside them in (a) and (c). 


\section{CO-RELATION BETWEEN WRINKLES AND BUBBLES}
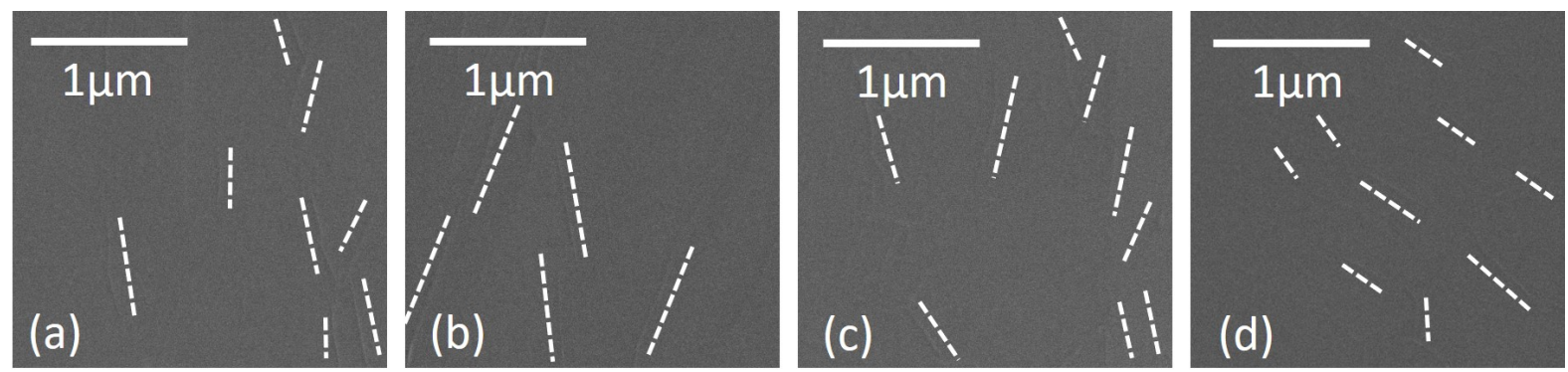

Fig. S 6: SEM images of graphene wrinkles under different treatment conditions. (a) Before heat-treatment; (b) after heat-treatment; (c) introduced into liquid nitrogen before heat treatment; (c) introduced into liquid nitrogen after heat treatment.
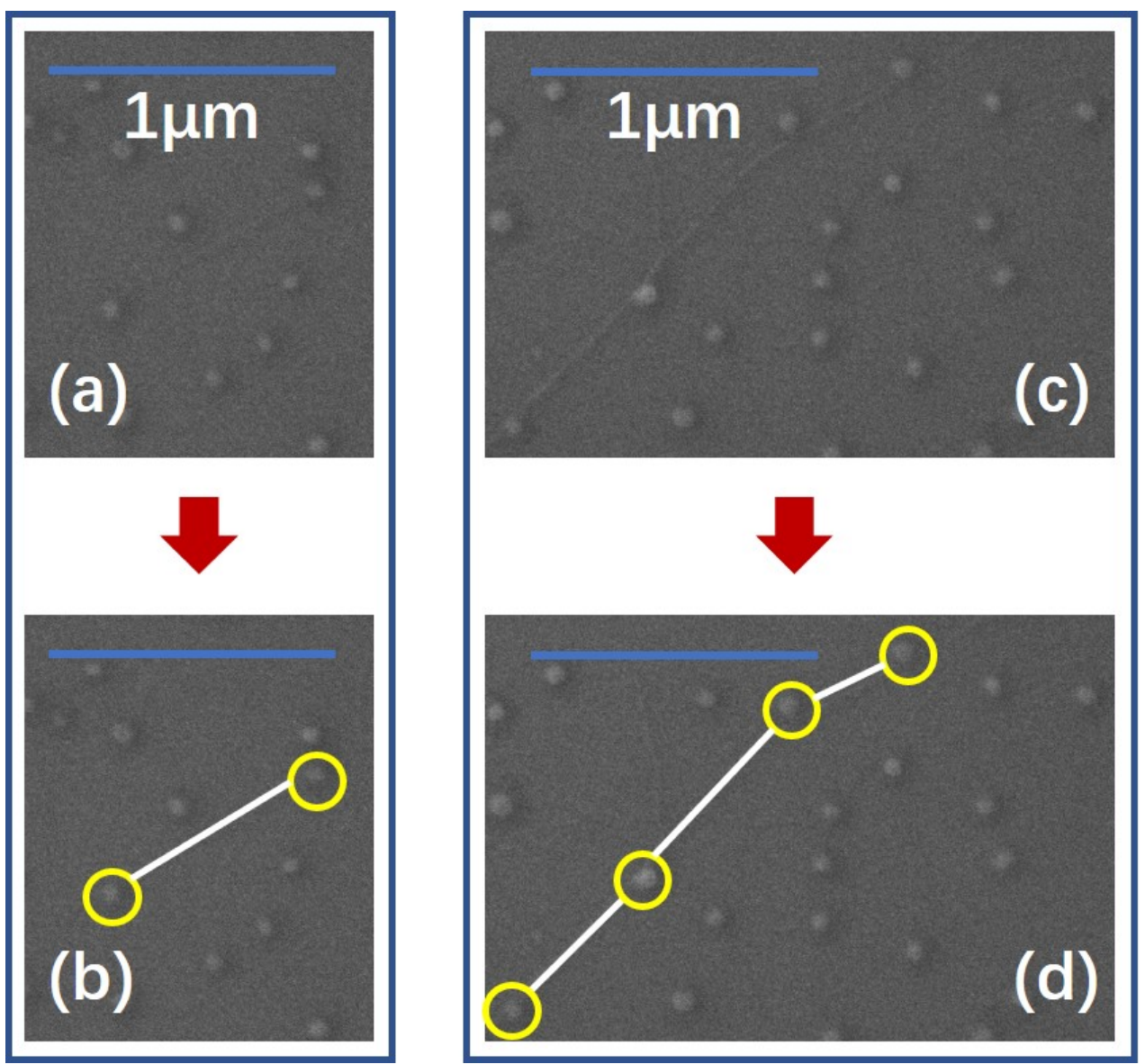

Fig. S 7: SEM images of graphene wrinkles and bubbles. The bubbles and wrinkles are marked with yellow circles and white lines respectively in (b) and (d). 


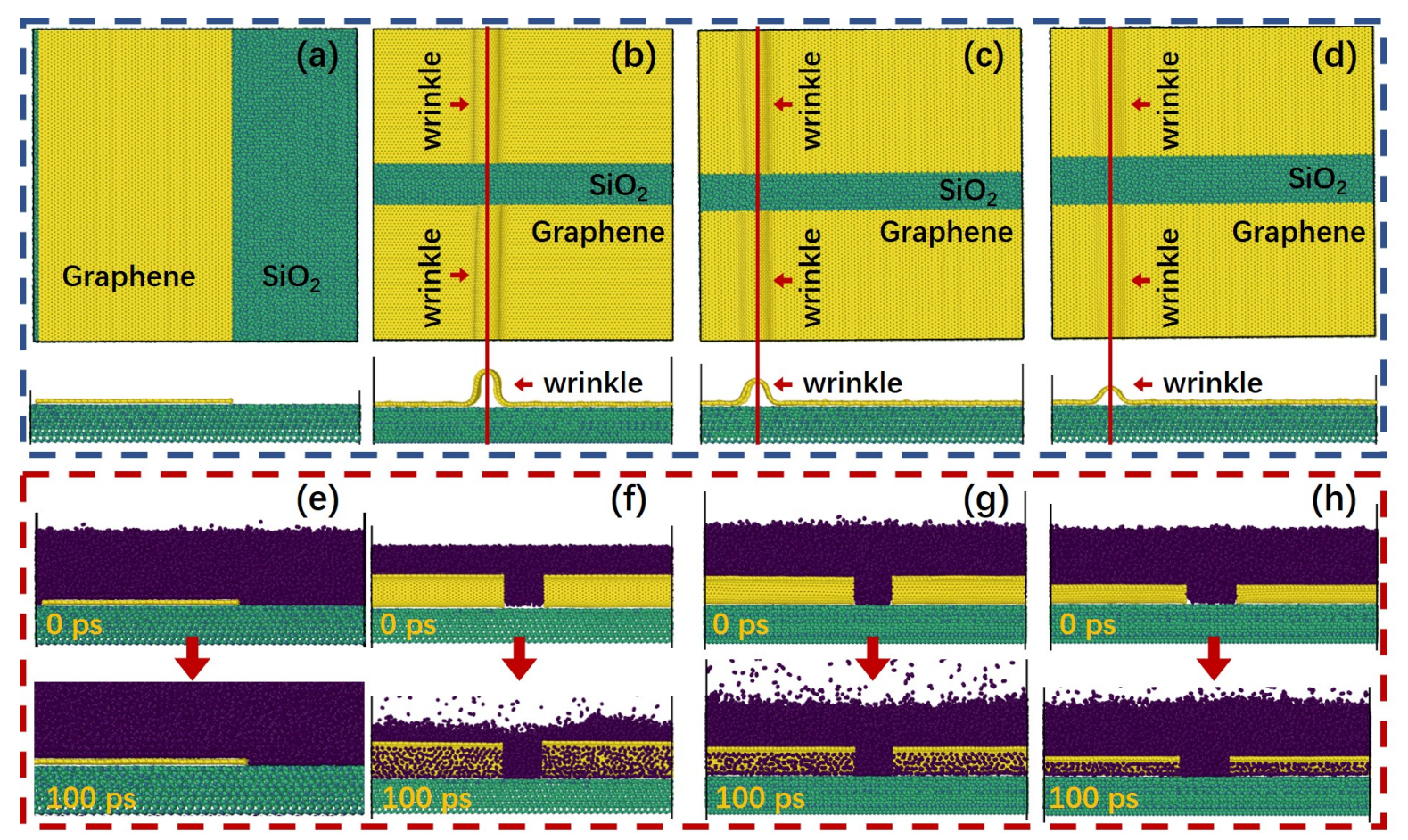

Fig. S 8: Upper panel: (a) graphene without wrinkles; (b), (c) and (d) graphene wrinkles with three different size. Lower panel: (e) snap shots of the diffusion behavior of liquid nitrogen at the edge of graphene without wrinkles; (f), (g) and (h) snap shots of the diffusion behavior of liquid nitrogen in the graphene wrinkles. (f), (g) and (h) show the cross-sectional view of the wrinkles. 


\section{PEAKS SHIFT}

In order to show the peak shift of Fig. 6a more distinctly, the peak positions of all the peaks are extracted, and the relationships between the peak position and the number of nitrogen molecule are plotted in Fig. S9. It can be clearly seen that as the number of nitrogen molecules increases, the peak values increases accordingly.

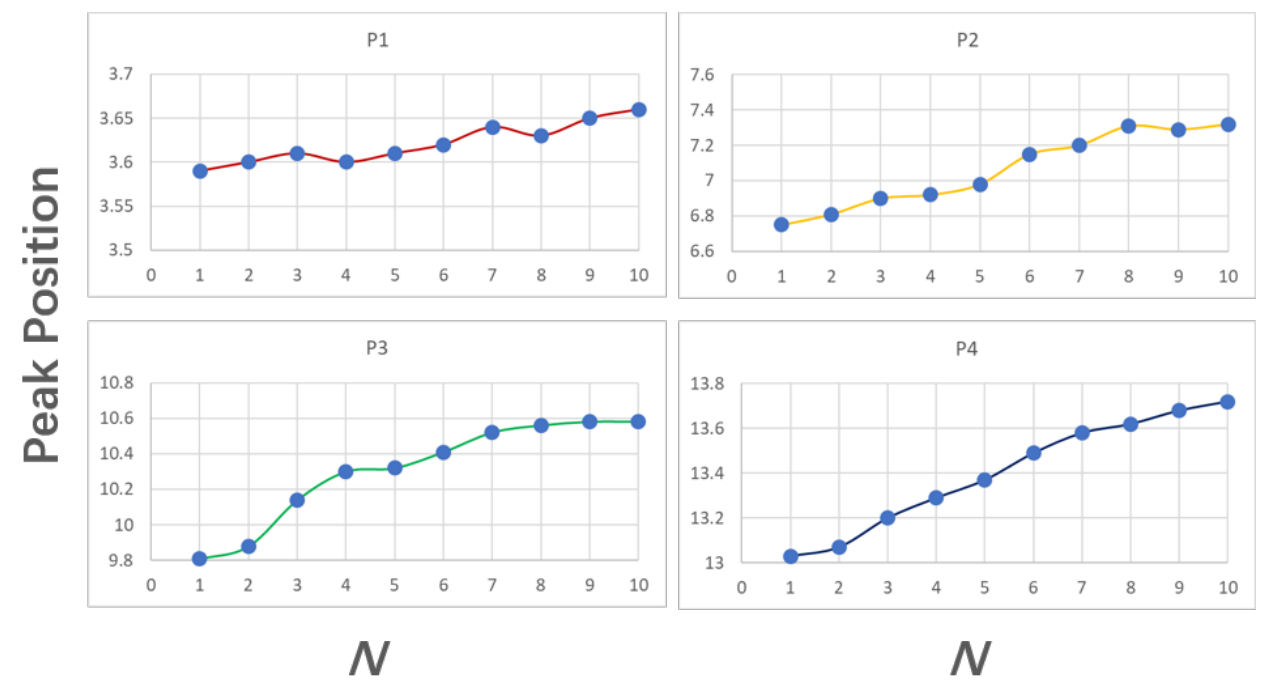

Fig. S 9: The relationships between the peak position and the number of nitrogen molecule.

In order to show the peak shift of Fig. $6 \mathrm{~d}$ more distinctly, the positions of each peak are connected with black lines (Fig. Sio), and it can be clearly seen that the lines are not perpendicular to the horizontal axis and the slope of them are negative. In this way we can clearly show the peaks shift.

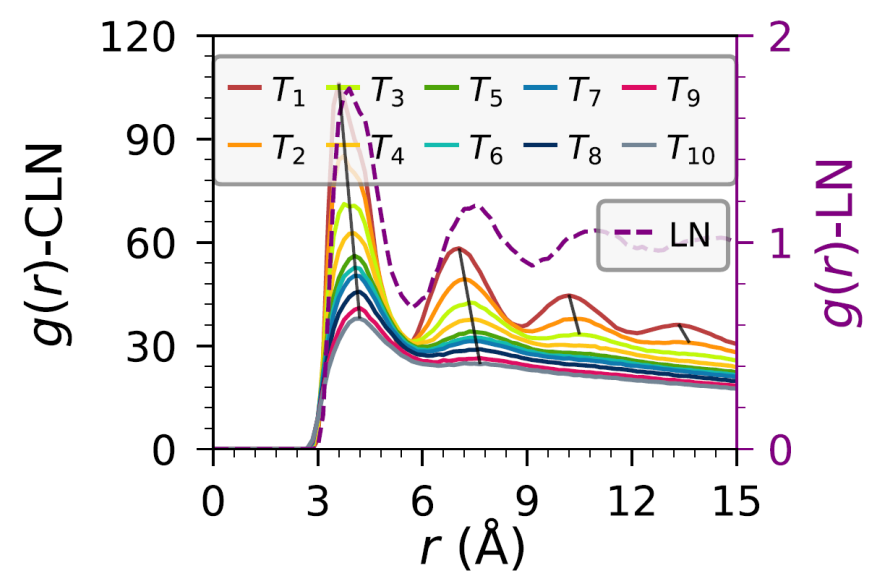

Fig. S 10: The correlation between temperature and radial distribution functions (RDFs). The black lines connect the positions of each peak.

\section{PHASE TRANSITION OF NITROGEN MOLECULES}

In order to figure out the phase transition process of nitrogen molecules, we cited the phase diagram of nitrogen (Fig. S11a).[1] According to the results of molecular dynamics simulation, it is found that as 
the temperature increases, the pressure of nitrogen inside the bubbles change very little (see Fig. Si1b below or Fig. $5 \mathrm{~h}$ in our manuscript). According to the values of temperature and pressure, we marked the state of nitrogen at different temperatures $\left(T_{1}, T_{2}, T_{3}\right.$ and $\left.T_{4}\right)$ with purple solid circles in Fig. Sira. It is found that when the temperature is equal to $T_{1}$, the nitrogen is in a liquid phase. As the temperature increases, nitrogen gradually changes from liquid phase to gaseous phase.
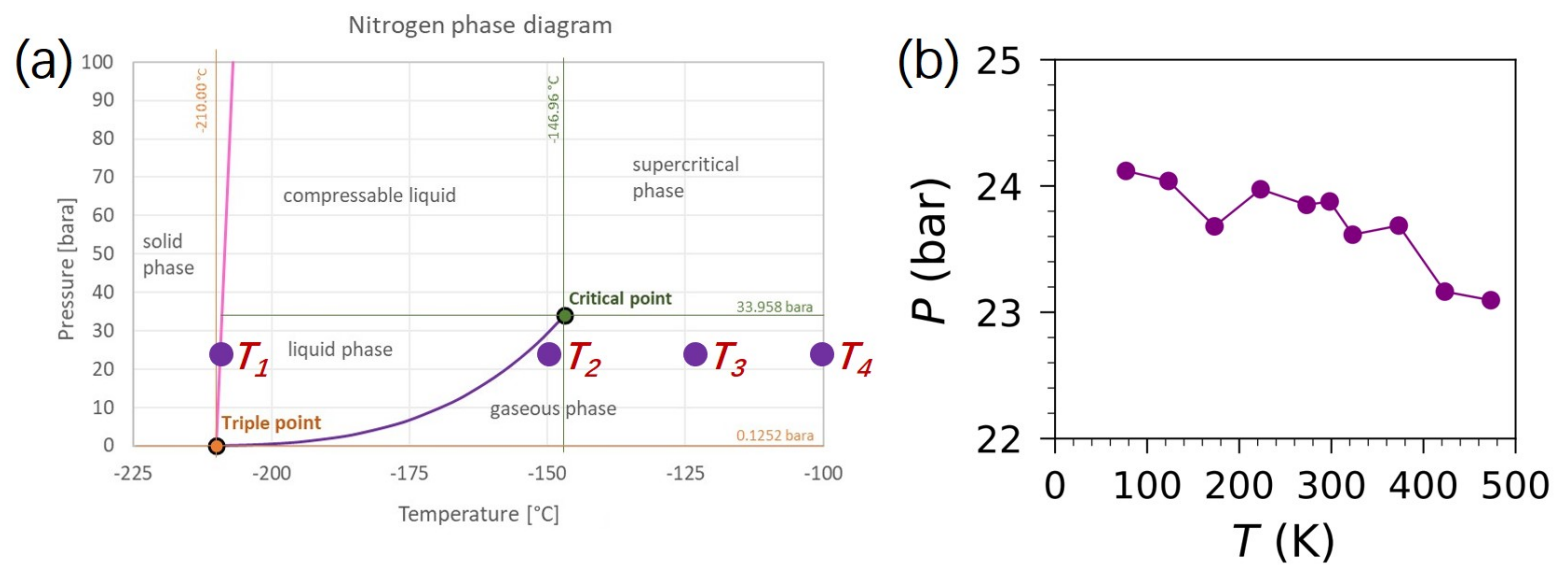

Fig. S 11: (a) Phase diagram of nitrogen; (b) pressure inside the bubbles at different temperatures.

In order to analyze the movement state of the nitrogen molecules more clearly, the snap shots of the molecule states and the molecular trajectories at different temperatures are illustrated in the upper and lower panel of Fig. S12 respectively. As the temperature increases, it is found that the range of motion of the nitrogen molecules will gradually expand, and the configuration would become more disordered (upper panel of Fig. S12). The molecular trajectory diagram (lower panel of Fig. S12) also clearly shows that as the temperature increases, the range of molecular motion gradually expands and the trajectory becomes more chaotic. In our work, we used diffusion coefficient to analyze the phase of nitrogen. By

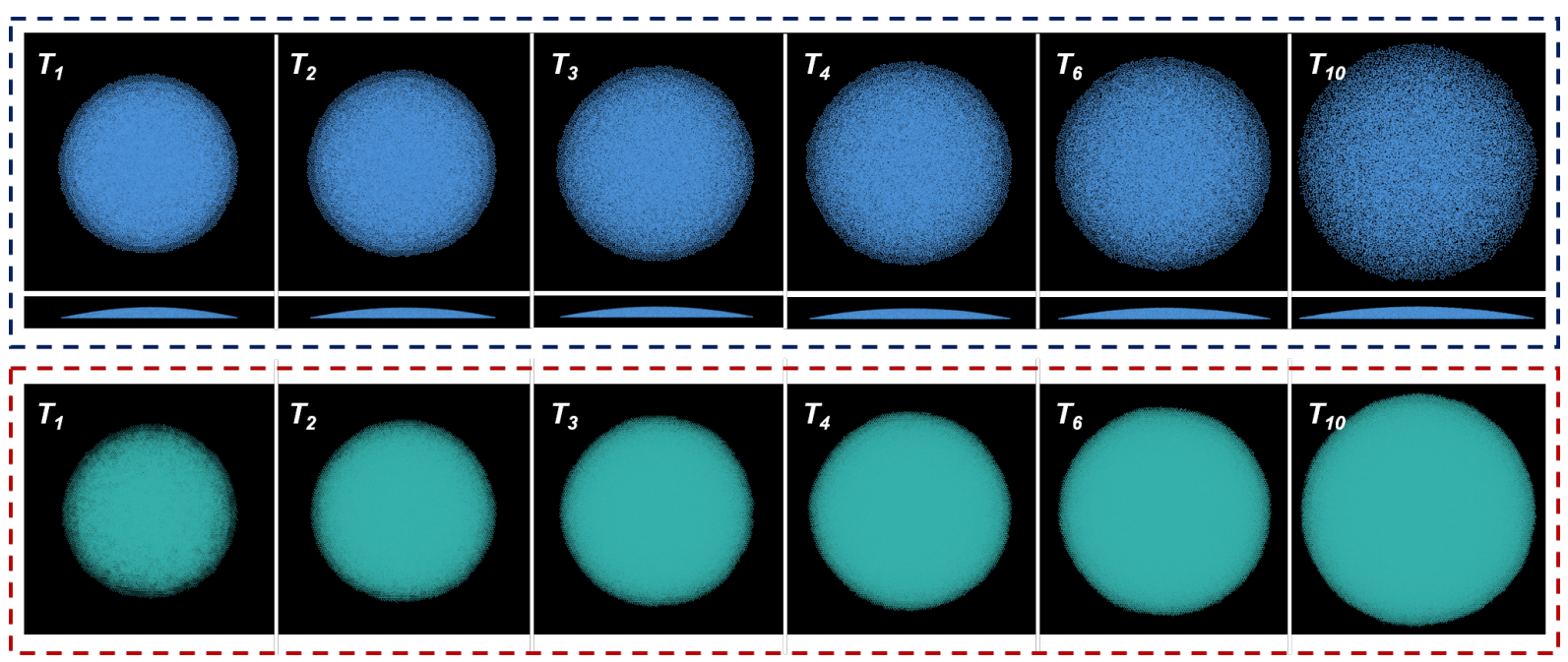

Fig. S 12: Upper panel: the snap shots of the molecule states at each temperature; Lower Panel: molecular trajectories of the nitrogen molecules at each temperature.

comparing with liquid nitrogen, we have initially judged the states of the confined nitrogen molecules based on the diffusion coefficients, which is actually incomplete. A more reasonable method is to combine the phase diagram of nitrogen to judge the phase of nitrogen molecules. As shown in Fig. Si1a, when the temperature over $150 \mathrm{~K}$, the nitrogen molecules is in a gaseous phase and no longer liquid. 
To build a realistic $\mathrm{SiO}_{2}$ substrate, we follow the method of Pellenq et al[2]. The $\beta$-cristobalite $\mathrm{SiO}_{2}$ crystal is cleaved along the (111) crystallographic face and the surface is terminated by oxygen atoms. The obtained substrate is a rectangular domain of $800 \AA \times 800 \AA \times 21 \AA$ with periodic boundary conditions in $x$ and $y$ directions. The bottom with $4 \AA$ of the substrate is fixed during the simulation procedure. The initial configuration of LN which contains specific number of nitrogen molecules is cylinder. The size of monolayer graphene membrane is equal to the dimension of $\mathrm{SiO}_{2}$ substrate, i.e. $800 \AA \times 800 \AA$.

To describe the interaction among carbon atoms, we use the adaptive intermolecular reactive empirical bond order (AIREBO)[3]. To model the $\mathrm{Si}-\mathrm{Si}, \mathrm{O}-\mathrm{O}$, and $\mathrm{Si}-\mathrm{O}$ atomic interactions, we use the Tersoff-type potential parameterization of Si-O systems by Munetoh and co-workers[4]. Harmonic bond are employed to keep the $\mathrm{N} \equiv \mathrm{N}$ distance close to $1.1 \AA$. The Si-C, O-C, Si-N, O-N, C-N and N-N interactions are described by the Lennard-Jones (L-J) potential function, written as

$$
V(r)=4 \epsilon\left[\left(\frac{\sigma}{r}\right)^{12}-\left(\frac{\sigma}{r}\right)^{6}\right],
$$

where $\epsilon$ is the energy parameter which determine the depth of the potential well; $\sigma$ is the distance parameters which determine the position of zero potential; $r$ is the distance between two atoms. The van der Waals (vdW) interaction parameters used in our simulations are based on the universal force filed (UFF) [5] and the detailed parameters are listed in Table $S_{1}$ and Table S2. The cutoff distance of the L-J potential is $18 \AA$. All cross terms are calculated by the Lorentz-Berthelot mixing rules[6]. We implement all MD simulations using the Large-scale Atomic/Molecular Massively Parallel Simulator (LAMMPS; https://lammps.sandia.gov/) package[7].

For the quantum chemistry calculations, we use Gaussian 16 (Revision Bo1) software package[8] to produce the wave function file of the $2 \mathrm{D}$ solid nitrogen. The single point energy calculation is performed by the functional B97D with Grimmes DFT-D 3 dispersion correction using the Becke-Johnson damping function[9, 10], and the def2-TZVP basis set is adopted[11]. The NCI analysis are carried out by using the Multiwfn software[12], and the isosurface maps are rendered by the VMD 1.9.2 program[13] based on the outputs of Multiwfn.

Table. S 1: The van der Waals (vdW) interaction parameters used in the MD simulations

\begin{tabular}{llllllllll}
\hline & Si-Si & O-O & C-C & N-N & Si-C & Si-N & O-C & O-N & C-N \\
\hline$\epsilon(\mathrm{kcal} / \mathrm{mol})$ & 0.402 & 0.060 & 0.105 & 0.069 & 0.206 & 0.167 & 0.079 & 0.064 & 0.085 \\
\hline$\sigma(\AA)$ & 3.822 & 3.118 & 3.430 & 3.261 & 3.623 & 3.542 & 3.274 & 3.190 & 3.345 \\
\hline
\end{tabular}

11

\section{DIFFUSION COEFFICIENT}

The average mean square displacement $(M S D)$ is defined as

$$
M S D=\frac{1}{N} \sum_{i=1}^{N}\left\langle\left[\vec{r}_{i}\left(t+t_{0}\right)\right]^{2}-\left[\vec{r}_{i}\left(t_{0}\right)\right]^{2}\right\rangle,
$$

where $N$ is the total number of nitrogen atoms, and $\vec{r}_{i}(t)$ is the displacement of $i$-th nitrogen atom at time $t$. The diffusion coefficient is defined as

$$
D=\lim _{t \rightarrow \infty} \frac{1}{2 d t} \times M S D,
$$

where $d$ equals to the dimension of the simulation system[14]. 


\section{PHASE TRANSITION BARRIER}

The Arrhenius equation can be written as

$$
D=D_{0} \exp \left(-\frac{E_{t}}{k_{B} T}\right),
$$

where $D$ is the diffusion coefficient, $T$ is the temperature, $E_{t}$ the phase transition barrier, and $k_{B}$ is the Boltzmann constant. Equation 4 can be transformed to

$$
\ln D=-\frac{E_{t}}{1000 k_{B}} \frac{1000}{T}+\ln D_{0} .
$$

Therefore, $E_{t}$ is calculated by a linear fitting to the dependence of $\ln D$ over $1000 / T$ [14].

\section{DESCRIPTIONS OF THE SUPPLEMENTARY MOVIES}

- MD_o.mp4: the whole simulation process

- MD_1.mp4: the height change of graphene film during the dynamical equilibrium of the confined nitrogen (top view)

- wfo_ln.mp4: diffusion behavior of the liquid nitrogen at the edge of graphene film without wrinkles

- wf1_ln.mp4 to wf3_ln.mp4: diffusion behavior of the liquid nitrogen in the graphene wrinkles with three different size

- T1.mp4 to T1o.mp4: kinetic behavior of the nitrogen molecules at different temperatures $\left(T_{1}=\right.$ $77 \mathrm{~K} ; \mathrm{T}_{2}=123 \mathrm{~K} ; \mathrm{T}_{3}=150 \mathrm{~K} ; \mathrm{T}_{4}=173 \mathrm{~K} ; \mathrm{T}_{6}=298 \mathrm{~K} ; \mathrm{T}_{10}=473 \mathrm{~K}$.) 


\section{REFERENCES}

[1] Engineering ToolBox (2008). Nitrogen-thermophysical properties [online]. https://www. engineeringtoolbox.com/nitrogen-d_1421. html [Accessed October 25, 2020].

[2] Joël Puibasset and Roland J.-M. Pellenq. Grand canonical monte carlo simulation study of water structure on hydrophilic mesoporous and plane silica substrates. The Journal of Chemical Physics, 119(17):9226-9232, 2003.

[3] Steven J. Stuart, Alan B. Tutein, and Judith A. Harrison. A reactive potential for hydrocarbons with intermolecular interactions. The Journal of Chemical Physics, 112(14):6472-6486, 2000.

[4] Interatomic potential for Si-O systems using tersoff parameterization. Computational Materials Science, 39(2):334-339, 2007.

[5] A. K. Rappe, C. J. Casewit, K. S. Colwell, W. A. Goddard, and W. M. Skiff. UFF, a full periodic table force field for molecular mechanics and molecular dynamics simulations. Journal of the American Chemical Society, 114(25):10024-10035, 1992.

[6] D. J. Tildesley M. P. Allen. Computer Simulation of Liquids. Oxford University Press, Oxford, 1989.

[7] Steve Plimpton. Fast parallel algorithms for short-range molecular dynamics. Journal of Computational Physics, 117(1):1 - 19, 1995.

[8] M. J. Frisch, G. W. Trucks, H. B. Schlegel, G. E. Scuseria, M. A. Robb, J. R. Cheeseman, G. Scalmani, V. Barone, G. A. Petersson, H. Nakatsuji, X. Li, M. Caricato, A. V. Marenich, J. Bloino, B. G. Janesko, R. Gomperts, B. Mennucci, H. P. Hratchian, J. V. Ortiz, A. F. Izmaylov, J. L. Sonnenberg, D. Williams-Young, F. Ding, F. Lipparini, F. Egidi, J. Goings, B. Peng, A. Petrone, T. Henderson, D. Ranasinghe, V. G. Zakrzewski, J. Gao, N. Rega, G. Zheng, W. Liang, M. Hada, M. Ehara, K. Toyota, R. Fukuda, J. Hasegawa, M. Ishida, T. Nakajima, Y. Honda, O. Kitao, H. Nakai, T. Vreven, K. Throssell, J. A. Montgomery, Jr., J. E. Peralta, F. Ogliaro, M. J. Bearpark, J. J. Heyd, E. N. Brothers, K. N. Kudin, V. N. Staroverov, T. A. Keith, R. Kobayashi, J. Normand, K. Raghavachari, A. P. Rendell, J. C. Burant, S. S. Iyengar, J. Tomasi, M. Cossi, J. M. Millam, M. Klene, C. Adamo, R. Cammi, J. W. Ochterski, R. L. Martin, K. Morokuma, O. Farkas, J. B. Foresman, and D. J. Fox. Gaussian 16 Revision B.01, 2016. Gaussian Inc. Wallingford CT.

[9] Stefan Grimme. Semiempirical gga-type density functional constructed with a long-range dispersion correction. Journal of Computational Chemistry, 27(15):1787-1799, 2006.

[10] Stefan Grimme, Stephan Ehrlich, and Lars Goerigk. Effect of the damping function in dispersion corrected density functional theory. Journal of Computational Chemistry, 32(7):1456-1465, 2011.

[11] Florian Weigend and Reinhart Ahlrichs. Balanced basis sets of split valence, triple zeta valence and quadruple zeta valence quality for $\mathrm{H}$ to Rn: Design and assessment of accuracy. Phys. Chem. Chem. Phys., 7:3297-3305, 2005.

[12] Tian Lu and Feiwu Chen. Multiwfn: A multifunctional wavefunction analyzer. Journal of Computational Chemistry, 33(5):580-592, 2012.

[13] William Humphrey, Andrew Dalke, and Klaus Schulten. Vmd: Visual molecular dynamics. Journal of Molecular Graphics, 14(1):33 - 38, 1996.

[14] Yifei Mo, Shyue Ping Ong, and Gerbrand Ceder. First principles study of the $\mathrm{Li}_{10} \mathrm{GeP}_{2} \mathrm{~S}_{12}$ lithium super ionic conductor material. Chemistry of Materials, 24(1):15-17, 2012. 